\title{
Functional MRI Applications in Epilepsy Surgery
}

\author{
Craig A. Beers, Paolo Federico
}

\begin{abstract}
Functional magnetic resonance imaging (fMRI) is a non-invasive neuroimaging technique that has grown rapidly in popularity over the past decade. It is already prevalent in psychology, cognitive and basic neuroscience research and is being used increasingly as a tool for clinical decisionmaking in epilepsy. It has been used to determine language location and laterality in patients, sometimes eliminating the need for invasive tests. fMRI can been used pre-surgically to guide resection margins, preserving eloquent cortex. Other fMRI paradigms assessing memory, visual and somatosensory systems have limited clinical applications currently, but show great promise. Simultaneous recording of electroencephalogram (EEG) and fMRI has also provided insights into the networks underlying seizure generation and is increasingly being used in epilepsy centres. In this review, we present some of the current clinical applications for fMRI in the pre-surgical assessment of epilepsy patients, and examine a number of new techniques that may soon become clinically relevant.
\end{abstract}

\begin{abstract}
RÉSUMÉ: Applications de l'IRM fonctionnelle dans la chirurgie de l'épilepsie. L'imagerie par résonance magnétique fonctionnelle (IRMf) est une technique de neuroimagerie non effractive dont la popularité s'est accrue rapidement au cours des dix dernières années. Son utilisation est courante en psychologie et en recherche fondamentale en neurosciences, et elle est de plus en plus utilisée comme instrument de décision clinique dans l'épilepsie. Elle a été utilisée pour déterminer la zone du langage et la latéralité chez certains patients, évitant ainsi parfois d'avoir recours à des tests effractifs. L'IRMf peut être utilisée avant la chirurgie pour aider à déterminer les marges de résection, permettant ainsi de préserver des fonctions corticales importantes. D'autres paradigmes d'utilisation de l'IRMf pour évaluer la mémoire et les systèmes visuel et somatosensitifs ont actuellement des applications cliniques limitées mais ils sont très prometteurs. L'enregistrement électroencéphalographique et l'IRMf simultanés ont également fourni des informations sur les réseaux qui sous-tendent la génération des crises et sont de plus en plus utilisés dans les centres de traitement de l'épilepsie. Dans cette revue, nous présentons certaines des applications cliniques actuelles de l'IRMf dans l'évaluation préopératoire des patients atteints d'épilepsie et nous discutons de quelques techniques nouvelles qui pourraient bientôt devenir intéressantes au point de vue clinique.
\end{abstract}

Can. J. Neurol. Sci. 2012; 39: 271-285

Epilepsy surgery is a potentially life-altering procedure that is typically reserved for patients with medically intractable epilepsy ${ }^{1}$. This sub-population accounts for $20-40 \%$ of all persons diagnosed with epilepsy; their surgical outcomes vary greatly and are highly dependent on the types of surgeries performed $^{2,3}$. Accurate identification of the region of cortex that generates seizure activity (the seizure focus or ictal onset zone) is essential in the pre-surgical assessment of epilepsy patients ${ }^{4,5}$. Determination of the ictal onset zone is typically accomplished by video-electroenephalogram (EEG) monitoring (VEM), structural magnetic resonance imaging (MRI), single photon emission computerized tomography (SPECT) and positron emission tomography (PET).

Video-EEG monitoring involves the simultaneous recording of EEG and observable patient behaviour ${ }^{6}$. Concurrent observation of ictal EEG and behavioural manifestations, when examined by trained personnel, provides important localization information ${ }^{6}$. Since its introduction in the early 1980s, MRI has quickly replaced computed tomography (CT) scanning as the imaging modality of choice for epilepsy patients ${ }^{7}$. Magnetic resonance images are vital in the identification of epileptogenic foci of diverse etiologies (e.g., malformations of cortical development, hippocampal sclerosis, or cerebral cavernomas, etc. $)^{7-9}$. For patients without observable structural abnormalities, functional imaging such as SPECT and positron emission tomography (PET) may aid in localization. Ictal SPECT involves the intravenous injection of a radioactive tracer [such as technetium- $99 \mathrm{~m}$ hexamethylpropylene amine oxime $\left({ }^{99} \mathrm{mTc}-\right.$ HMPAO)] as quickly as possible following seizure onset ${ }^{10,11}$. The tracers allow mapping of local ictal increases in cerebral perfusion, which correlate strongly with epileptogenic foci ${ }^{7,10,12}$.

From the Hotchkiss Brain Institute (CAB, PF), Seaman Family MR Research Centre

(CAB, PF); Departments of Clinical Neurosciences(CAB, PF) and Radiology (PF),

University of Calgary, Calgary, Alberta, Canada.

Received July 13, 2011. Final Revisions Submitted December 5, 2011. Correspondence to: Paolo Federico, Departments of Clinical Neurosciences and Radiology, University of Calgary, Room C1214a, Foothills Medical Centre, 1403 29th Street NW, Calgary, Alberta, T2N 2T9, Canada. Email: pfederic@ucalgary.ca 
Interictal SPECT is also used routinely, where hypoperfusion is seen in potentially epileptogenic regions ${ }^{13-15}$. Positron emission tomography studies utilize radioactive glucose molecules such as 2-[18F]fluoro-2-deoxyglucose to image cerebral metabolism. However, due to the dynamics of cerebral metabolism, a PET tracer injection requires several minutes of circulation before reliable results may be achieved ${ }^{12,16}$. As such, PET scans are typically performed interictally (not ictally), looking for areas of hypometabolism indicative of epileptogenic tissue ${ }^{16}$.

Though less commonly used, magnetoencephalography (MEG) and transcranial magnetic stimulation (TMS) may also be employed for pre-surgical assessment ${ }^{17-20}$. MEG measures local magnetic fields induced primarily by postsynaptic activity in the pyramidal cells of the cerebral cortex ${ }^{17,19}$. Unlike EEG, MEG is not distorted by signals from the skull and tissues surrounding the brain and can be quite effective at localizing the epileptic focus, particularly when it is located in superficial areas of the brain. However, MEG availability is currently limited due to the high cost of equipment and setup ( $>$ \$2 million USD) compared to a good quality VEM system (\$30-35000 USD) $)^{17}$. Transcranial magnetic stimulation uses magnetic stimulation to generate very local electric current in the cerebral cortex ${ }^{20}$. This current induces ion flow and causes neuronal activity to increase at the region of interest. The use of TMS pre-surgically typically involves mapping of the epileptogenic focus and surrounding eloquent cortex. Though TMS mapping carries a low risk for adverse side effects, it has been shown to induce unintentional seizure activity and raise cortical excitability ${ }^{20}$.

A limitation of PET, interictal SPECT, MEG, and TMS is that they rely on interictal rather than ictal measurements. While interictal spiking is usually indicative of epileptogenic tissue, concordance between ictal and interictal zones is not always present and it can often be difficult to differentiate the epileptogenic zone from sites of discharge propagation ${ }^{21-23}$.

Additional assessments may be performed to help determine eloquent cortex, which is to be avoided surgically. Neuropsychological testing is recommended in most cases and encompasses a battery of tests examining memory, handedness, and verbal fluency amongst other functions ${ }^{24}$. These tests establish a baseline of function, help detect pre-surgical deficits, and may predict post-surgical losses. In cases where the epileptogenic focus may border visual cortex, pre-operative visual field mapping is often performed ${ }^{25}$.

Functional MRI (fMRI) measures brain activity noninvasively and is still a relatively new technology that has great potential to aid in pre-surgical assessment of patients with epilepsy. As we will discuss in this review, fMRI may aid in language and memory lateralization as well as motor, sensory, and visual field mapping. In addition, combining EEG-fMRI may also help identify the seizure focus.

\section{THE BOLD RESPONSE}

Functional MRI has established itself as a versatile tool in the assessment of patients with epilepsy since it was first described by $\mathrm{Ogawa}^{26}$. The most common fMRI technique detects blood flow-related changes in venous deoxyhemoglobin content, the so-called Blood Oxygenation Level Dependent (BOLD) contrast technique, to generate functional maps of neural activation and deactivation (see next paragraph) ${ }^{26-29}$. The BOLD response is generated when an increase in neuronal activity in a given area results in a decrease in local deoxyhemoglobin concentration, which produces a reduction in local magnetic field inhomogeneities. These changes are detected as an increase in signal intensity when the brain is imaged using specific MR sequences sensitive to these changes (e.g., gradient echo imaging).

The time course of BOLD signal changes is a result of activity-dependent alterations in blood flow, blood volume and oxygen metabolism, which creates a characteristic BOLD response curve (Figure 1) 28,30,31. Oxyhemoglobin levels typically drop initially due to an immediate increase in local oxygen metabolism and extraction, creating an 'initial dip' in the BOLD signal $^{32}$. Following this, there is an increase in local blood flow that exceeds metabolic requirements. As a result, there is a paradoxical decrease in the proportion of deoxyhemoglobin relative to oxyhemoglobin (e.g., "washout of deoxyhemoglobin"), which produces an increase in BOLD signal that usually peaks in three to seven seconds. The BOLD signal then returns to baseline over several seconds and may potentially dip temporarily below baseline creating a "post-stimulus undershoot $^{\prime 32}$. It is important to note that the BOLD signal does not directly measure neuronal activity; it measures a vascular response which is closely correlated with neuronal activation ${ }^{33}$.

Clinical fMRI studies, particularly those of language, motor and memory systems, provide quantitative data that can guide clinical decision-making. The primary statistical analysis of fMRI signal involves examining the BOLD dataset in a hypothesis-driven manner ${ }^{33}$. The brain is imaged in three dimensions, dividing it into small cubes known as voxels. Imaging matrices for clinical purposes vary, but they are typically between $32 \times 32$ and $128 \times 128$ voxels per slice; when 30 slices are collected per volume, data can be generated for nearly

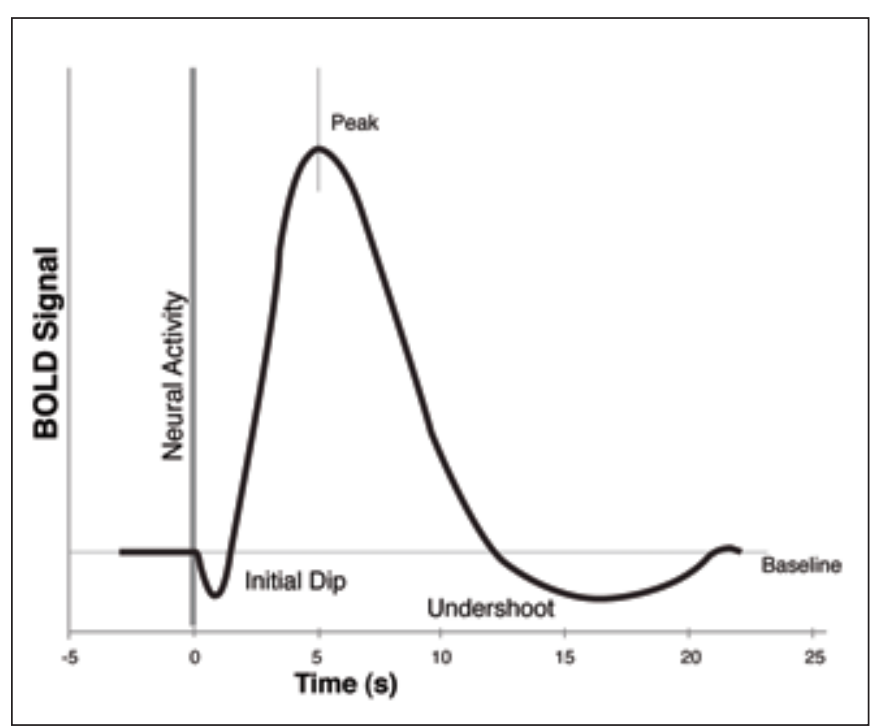

Figure 1: BOLD response. An example of a typical hemodynamic response during an fMRI scan. After an initial dip in BOLD signal at the onset of neural activity, a peak is seen after a delay of 3-7 seconds. An undershoot is typically seen before the signal returns to baseline. 
500000 distinct voxels. The BOLD signal measured for every voxel in the brain over the course of an fMRI study is then statistically compared to predicted time course for the BOLD signal for active voxels during the same scan, usually thresholded to $\mathrm{p}<0.05$ or greater ${ }^{33}$. For example, voxels showing increased BOLD signal during the task but not at rest are deemed active. Multiple trials of the same task are typically averaged over the course of an fMRI study to increase statistical power. Since there is great variability between subjects and scans, absolute peak activation values provide limited clinical information. Maps of the extent of activity and locations of maximal recorded BOLD signal, on the other hand, provide more valuable data. Functional MRI analyses may also be performed using a more data-driven method ${ }^{33}$, however, the majority of clinical assessments are currently hypothesis-driven.

\section{LANGUAGE fMRI}

Language representation in the brain encompasses a large neural network involving many sites in the frontal, parietal and temporal lobes ${ }^{34,35}$. Determining hemispheric language dominance prior to epilepsy surgery is essential to minimize post-operative cognitive deficits and may also be important for interpreting seizure symptomatology ${ }^{34-39}$. In the past, hemispheric dominance was determined using dichotic listening tests, sodium amobarbital testing (Wada test), or cortical stimulation.

\section{Dichotic Listening Test}

The dichotic listening test is a non-invasive behavioural task that involves the simultaneous presentation of words to both of the subject's ears ${ }^{40}$. Participants then report which of the two words they heard and the number of correct responses for each ear is then calculated. A right ear advantage is indicative of left hemispheric language dominance, while the opposite is true for left ear advantage ${ }^{40}$. While non-invasive and simple to perform, the dichotic listening test has limitations; the task may provide inaccurate results if a patient's seizure focus involves auditory cortex, and it has been found to be less accurate in detecting right and bilateral language dominance. It also requires astute auditory attention, making the task challenging for children and those with mental impairments ${ }^{41}$.

\section{Intracarotid Amobarbital Procedure (IAP)}

The intracarotid amobarbital procedure (IAP), first utilized in the 1960s, uses intracarotid injections of amobarbital to selectively produce hemispheric loss of function ${ }^{42}$. When the injected sodium amobarbital reaches the hemisphere dominant for language, global aphasia is observed for 30 seconds to two minutes, allowing the determination of hemispheric language dominance by language testing performed at the same time ${ }^{42}$. The IAP is considered by some to be the gold standard for testing language lateralization ${ }^{36,37,39}$. However, there are disadvantages to this technique: sodium amobarbital is currently not available in Canada, and injection of sodium amobarbital requires conventional catheter angiography and is therefore invasive $^{34,36,37}$. The IAP also carries a reported risk of up to $10 \%$ for serious complications such as stroke, encephalopathy, or seizures ${ }^{43}$. Though widely used in the past, IAP lacks testing parameter standardization ${ }^{36}$ and is reported by patients as being unsettling and uncomfortable ${ }^{34}$. Etomidate is a commonly used anaesthetic that has emerged as a viable alternative to sodium amobarbital as it is more widely available and longer acting ${ }^{44,45}$. Etomidate has advantages over sodium amobarbital including improved control over length of hemianaesthesia. However, language testing with etomidate remains an invasive procedure, requiring multiple highly trained personnel. It is currently being used in a limited number of centres. Like the IAP, etomidate is currently used primary for memory testing (rather than language testing) as robust, clinically relevant memory fMRI paradigms are not currently available (see later).

\section{Cortical Stimulation}

Cortical stimulation mapping is another technique that can be used to map language function ${ }^{46-49}$. It is currently considered the gold standard for identifying eloquent cortical areas, short of resection. This technique can be performed intraoperatively or at the bedside by passing current across pairs of electrodes placed on the cortical surface. Application of sufficient current will then either transiently activate underlying tissue (e.g., produce a motor or sensory response) or interfere with a given function (e.g., language $)^{47-49}$. This technique can therefore provide an immediate and first-hand map of language function. It is, however, an invasive test requiring intracranial EEG electrodes (for bedside testing) or an awake craniotomy (for intraoperative mapping) and has limited coverage as testing can only be performed at the locations of electrode placement.

\section{Language fMRI}

Functional MRI is quickly gaining acceptance as a tool for determining language dominance and mapping language areas prior to epilepsy surgery ${ }^{34,50,51}$. Functional MRI is less costly and much less invasive than the IAP or cortical stimulation ${ }^{35,51}$, and it can be performed without the presence of clinical personnel apart from an MR technologist. Language fMRI is easily repeatable, if necessary. Language fMRI can also play a role in the interpretation of neuropsychological findings. In cases of indeterminate language findings via neuropsychological tests, fMRI provides quantitative data to help interpret the neuropsychological data and guide therapies. In cases where seizure lateralization is difficult to determine, language fMRI and neuropsychology testing provide complementary data by helping lateralize language function, particularly in the presence of bilateral language dominance.

A language fMRI study is performed by continuously acquiring functional MR images while asking subjects to perform a given language task (the task condition). These images are contrasted with images acquired while at rest (the rest or control condition). This is known as a blocked or boxcar design as periods of activity are alternated with periods of rest creating blocks of activity. While these language tasks may be provided to the subject through auditory cues, they are most commonly delivered via a computer monitor and presentation device due to scanner noise ${ }^{34}$. Tasks used may include noun-verb generation (e.g., "generate verbs that can be performed with a chair"), lexical retrieval (e.g., "think of nouns beginning with the letter T") and semantic recognition (e.g., "press button 1 if this object 
can be found in the kitchen"). During the rest period, subjects may be shown a fixation object or a blank screen. The rest period is meant to characterize resting state function, but this can be difficult to control and it may introduce unwanted cognitive activation. ${ }^{35}$ An alternative to a rest period is a control task such as an auditory discrimination task ${ }^{35}$. A paradigm may then include at least two, four-minute blocks of the given tasks. For example, language fMRI performed at our centre uses two runs each of the noun-verb and lexical retrieval tasks given in a block design (27 seconds of task and 27 seconds of baseline alternated for five minutes). Other examples include blocked noun-verb generation followed by semantic recognition ${ }^{39}$ and semantic recognition contrasted with an auditory discrimination $\operatorname{task}^{52}$.

The paradigms and tasks used by individual epilepsy centres vary greatly as there exists no accepted, standardized technique for performing language fMRI for clinical purposes. It is clear however, that reliance on a single paradigm for all language fMRI studies should be discouraged as different tasks may be necessary, such as using a simpler task for developmentally delayed patients or children. More than one task should also be used for a given patient to ensure activation across language networks occurs and possibly to selectively assess receptive versus expressive activation ${ }^{38,52-54}$. A number of viable methods, varying in complexity are available. Currently, there are no published reports of specific language fMRI paradigms used clinical purposes across different centres. However, published reviews by Binder ${ }^{55}$ and Szaflarski et al $^{39}$ identify other language fMRI paradigms use for research purposes at other centres that might be used for clinical purposes.
Analysis of language fMRI involves the statistical comparison of brain activation (BOLD signal) during the language task to that during the rest (or control) task on a voxelby-voxel basis. Those voxels showing significantly greater BOLD signal changes during the task compared to rest (as determined by a t-test) are considered "activated" during the task. A colour scheme (reflecting statistical significance) is then applied to produce maps of fMRI activation ${ }^{39,51,56}$. These maps are typically overlaid onto anatomical images as shown in Figure 2. These maps of language activation can then be used to visually compare activation to known anatomical sites (e.g., Broca's area), known lesions (e.g., Figure 3), or a proposed target for surgical resection.

A laterality index (LI) can be calculated using language fMRI data, which provides a useful, quantitative measure of language lateralization $^{34,35,39,56}$. Though techniques vary, one common approach for measuring LI involves drawing regions of interest (ROIs) over the activated voxels in the left and right language areas (typically Broca's, Wernicke's and associated areas). The mean significance of activity within each ROI is then determined and compared between hemispheres to generate a value of the degree of lateralization of language-related activity. There are different techniques for the calculation of LI. The following is an example used in our epilepsy centre:

$$
\mathrm{LI}=\frac{(\mathrm{nL} \times \mathrm{sL})-(\mathrm{nR} \times \mathrm{sR})}{(\mathrm{nL} \times \mathrm{sL})+(\mathrm{nR} \times \mathrm{sR})}
$$

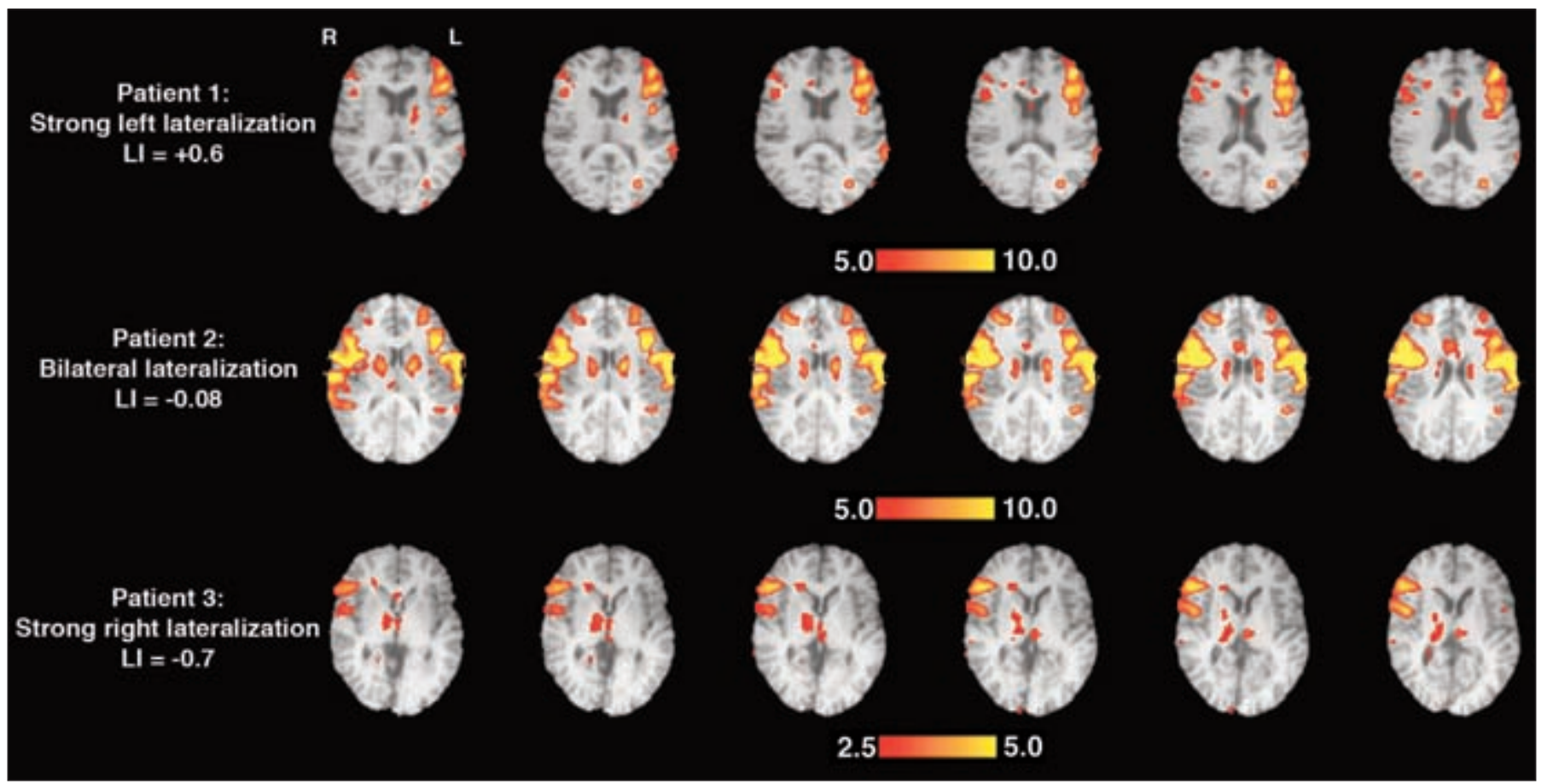

Figure 2: Language fMRI. Clinical language fMRI studies performed at our centre on three patients with epilepsy. Top Row: Patient with a left lateralized language dominance pattern with a laterality index $($ LI) of +0.6 . Middle Row: Patient with bilateral language representation $(L I=-0.08)$. Bottom Row: Patient with a right lateralized language representation $(L I=-0.7)$. 


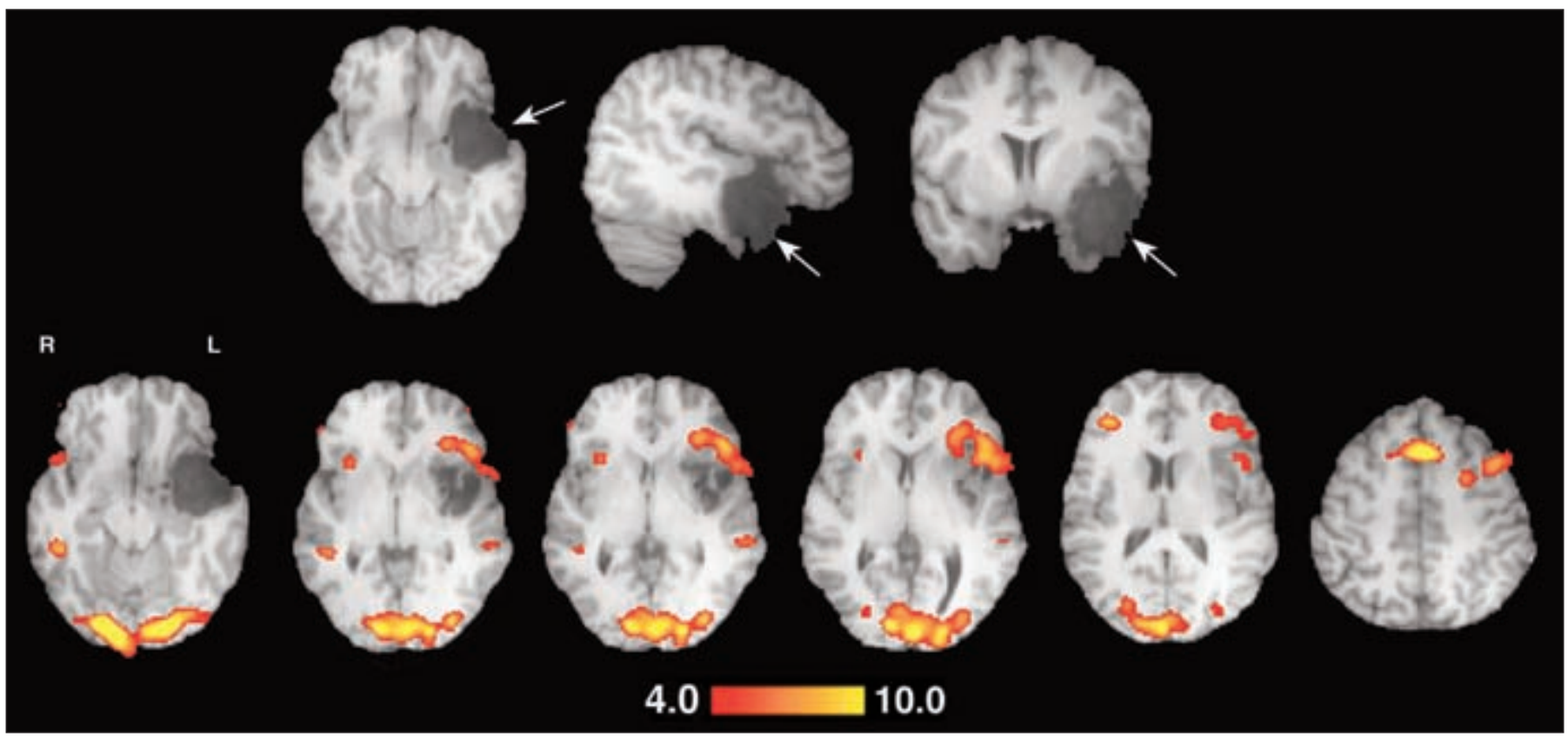

Figure 3: Language fMRI in a patient with epilepsy and a lesion. Top Row: Axial, saggital, and coronal T1-weighted images showing a lesion in the left anterior temporal lobe resembling cerebrospinal fluid, but not isointense to it. Bottom Row: Language fMRI activation appears in tissue surrounding the lesion but not within the lesion itself. The observed occipital BOLD signal reflects visual stimulation associated with visual presentation of language stimuli.

where $\mathrm{nL}$ and $\mathrm{nR}$ represent the total number of voxels in the left and right ROI, respectively, and sL and sR represent the mean significance of activity within left and right ROI, respectively. From this, it can be seen that an LI score of +1 suggests complete left lateralization and a score of -1 suggests complete right lateralization. Values between +0.2 and -0.2 indicate some degree of bilateral language representation, but these thresholds vary by centre. ${ }^{39}$ There are alternative methods for calculating LI (see ${ }^{55}$ and ${ }^{57}$ for example). In addition, an alternative to these types of calculations may be to assess the functional connectivity of the language areas during tasks ${ }^{58}$. Functional connectivity is discussed later in this review.

It is important to note that in the pre-operative assessment for an epileptic patient, language fMRI provides two different, but complementary datasets for the clinician: i) maps of language fMRI activation that can be visually compared to the proposed target of surgical resection, and ii) the LI, which provides information about language hemispheric dominance. This information is useful for the prediction and avoidance of postoperative deficits ${ }^{34,51}$. Indeed, several reports have shown a strong correlation between language lateralization results obtained through IAP and through fMRI, including 100\% concordance in an early published comparison ${ }^{59}$. Another study of 21 subjects found concordances of $91 \%$ (left dominant), $75 \%$ (mixed) and 67\% (right dominant) using a combined four-task language fMRI paradigm ${ }^{60}$. The same study also compared maps obtained from cortical stimulation to those obtained from language fMRI in the same patients. While only $51 \%$ of active voxels were confirmed by cortical stimulation, the absence of fMRI activation was $100 \%$ concordant with the absence of critical language function in 10 of 11 patients. The authors concluded that fMRI can reliably predict the absence of critical language areas, thus identifying areas that can be safely resected without the need for electrocortical stimulation mapping. While these data suggest that fMRI may eventually be used in place of cortical stimulation, definitive studies comparing the two modalities have not yet been published. Studies of patients with disorders other than epilepsy have provided some insight. For example, one review of nine published articles comparing the two modalities showed fMRI sensitivity and specificity values ranging from $59-100 \%$ and $0-97 \%$, respectively ${ }^{61}$. It was also found that language fMRI studies performed at higher field strengths (3.0T or greater) were more concordant with cortical stimulation then those performed at $1.5 \mathrm{~T}^{61}$.

The reproducibility of language fMRI is an important consideration. Language fMRI reproducibility has been previously assessed through a scan-rescan paradigm, testing the subjects on separate days ${ }^{62,63}$. Using a combined analysis of four language tasks on healthy controls ${ }^{62}$, the authors performed language fMRI scans a minimum of two months apart and compared the results. The laterality index was found to be reproducible between scans, but the absolute number of active voxels was not. A second study of patients with epilepsy showed that language fMRI can provide robust assessments of hemispheric dominance and language localization in clinical populations, however, the authors recommended further investigation to improve reliability ${ }^{63}$. Language fMRI reliability and reproducibility remains an active area of research as language tasks and testing paradigms are continually being created and their validity assessed. Some recent examples 
include the use of functionally defined language ROIs ${ }^{64}$ and new semantic tasks ${ }^{65,66}$.

Recently, language fMRI has been combined with diffusion tensor imaging (DTI) in an effort to examine the relationship between structure and function in the language system ${ }^{67}$. Diffusion tensor imaging provides measures of white matter tract organization and integrity. One study using combined fMRI-DTI showed that patients with right temporal lobe epilepsy who were left lateralized for language via fMRI had a higher fractional anisotropy (analogous with white matter tract strength and integrity) in the left (contralateral) arcuate fasciculus ${ }^{67}$. This relationship was not observed in patients with left temporal lobe epilepsy, leading the authors to suggest a decoupling between structural and functional lateralization indices in patients with left temporal lobe epilepsy. This novel technique continues to develop.

The block experimental design involves alternating periods of task-on and task-off conditions (discussed previously) and is commonly used for clinical language studies. However, eventrelated designs are now seen in the majority of newly published studies. In an event-related design, it is assumed that neural activity is a short and discrete event that evokes transient BOLD response. The timing and duration of these events are then used to generate an expected hemodynamic response for the entire dataset. The measured time-varying signal from each voxel in the brain is statistically compared to this modelled hemodynamic response function for the given task. A greater correlation with the modelled hemodynamic response function suggests a greater likelihood that a particular voxel was activated during the event of interest (e.g., language task). The degree of statistical correlation is then color-coded and displayed onto an anatomical image.

There are several advantages of using event related designs. Unlike block designs, event-related designs do not assume steady-state activation during a given task and treat each event as a singular occurrence, allowing the study of frequently occurring events. Event-related studies also tend to be more flexible in terms of design choices, are less sensitive to head motion, and allow for individual variations of the hemodynamic response ${ }^{68}$. On the other hand, event-related designs are more difficult to implement and analyze, potentially limiting clinical applications, especially with subjects who are unable to follow complex commands. Indeed, there are no published data using eventrelated designs for language assessment in epilepsy patients. However, one study compared the maps of language activation between six healthy controls and eight patients with brain tumors, as determined by block and by event-related designs ${ }^{68}$. The authors found similar, but not completely concordant language activation and suggest that event-related designs provide more robust activity, accounting for the increase in number of active voxels seen with that design.

Limitations and drawbacks remain in using fMRI in preoperative language assessment. First, language fMRI is not an inactivation test such as cortical stimulation or IAP. Hence, it is not possible to fully determine whether a region showing BOLD activation is truly critical for language or not. However, it is generally safe to resect regions that do not show activation as described above ${ }^{69}$. Another limitation relates to the lack of a standardized language protocols, scanners, and analytical techniques. In addition, validation of language fMRI has not been performed comprehensively in subjects with mixed dominance patterns, or in patients who show reorganization of language areas as a result of epilepsy or early brain insults ${ }^{34}$. Occasionally, subjects will show atypical language activation or no activation at all. In such cases, language fMRI can be repeated without harming the patient. Alternative language assessment methods may also be considered for clinical decision-making in these patients. Lastly, it is important to note that most language fMRI studies have small numbers of patients. It would therefore be ideal to perform systemic meta-analyses using similar patient types and tasks in order to make more definite conclusions in the field of language fMRI. A comprehensive meta-review of language fMRI research has been published in 2006 and provided significant insight into the language networks of the left hemisphere, but it did not address clinical applications nor did it address the heterogeneity of language tasks ${ }^{70}$. Despite these limitations, language fMRI has had a significant impact in epilepsy surgery programs for determination of language hemispheric dominance and, to a lesser extent, language localization.

\section{MEMORY fMRI}

Temporal lobe epilepsy is the most common form of refractory focal epilepsy in adults ${ }^{71-73}$. Surgical resection, most commonly an anterior temporal lobectomy, can provide seizure freedom in $60-90 \%$ of cases ${ }^{52,74-76}$. A complication of such surgery is memory decline, particularly verbal memory if the dominant temporal lobe is resected ${ }^{52,77}$. This complication arises due to the removal of the anterior hippocampal and parahippocampal regions, which are essential for many memory processes $^{52}$.

\section{Traditional Memory Testing}

Given the above, it is essential to evaluate memory prior to temporal lobe resection in order to identify pre-surgical memory deficits, and to predict postoperative memory outcomes ${ }^{76}$. In this context, neuropsychological testing and structural MRI are vital, while the IAP is used, albeit less frequently, to evaluate patients with atypical or ambiguous neuropsychological results $52,75,76,78$.

Neuropsychological testing of memory has proven itself as a strong predictor of memory outcome postoperatively in numerous trials ${ }^{76,78,79}$, and is a standard procedure in the preoperative assessment of patients with temporal lobe epilepsy. Though valuable, neuropsychological testing is time consuming, costly, and is less reliable in low functioning and non-verbal patients. The ability of structural MRI in predicting post-surgical memory decline is limited to cases where hippocampal sclerosis is clearly identified; hippocampal volumetric data has shown that larger hippocampal volumes on the resected side correlate strongly with a greater post-operative memory decline ${ }^{78,80}$. The role of high field structural MRI (3T or greater) and tractography (visualization of white matter tracts) in predicting memory decline after resection has not been adequately evaluated to date.

The goal of the IAP for memory is to test for adequate and inadequate memory function (both verbal and non-verbal material) in each hemisphere, in an effort to predict post-surgical outcomes. A review of the literature showed that $30-60 \%$ of patients with relatively stronger memory function lateralized to the side of surgery show poorer memory outcomes than patients 
whose memory function is greater on the side contralateral to the seizure focus ${ }^{52}$. The authors also cite inconsistent results of the IAP as a predictor of postoperative memory decline among published studies and highlight the questions that many authors have regarding the test-retest reliability and specificity of IAP ${ }^{52}$. This has led to some skepticism regarding this form of memory testing.

\section{Memory fMRI}

The IAP is traditionally considered to be the gold standard for determining hemispheric memory function. However, it is an invasive procedure with the risks described earlier. Since fMRI does not suffer from these limitations, memory fMRI is increasingly being utilized in the pre-operative assessment of patients undergoing temporal lobe resections. Published reports have detailed the ability of memory and language tasks performed during fMRI scans to predict memory decline post temporal lobe surgery $52,75,78,79,81,82$. Furthermore, a study of 60 patients undergoing anterior temporal lobe resections showed that fMRI is useful for identifying patients at risk for postoperative memory decline, while the IAP was less effective $^{52}$. The authors found that a calculation of the pre- and post-operative language laterality index using fMRI was a stronger predictor of postoperative memory decline $(r=-0.432$, $\mathrm{p}<.001)$ than IAP $(\mathrm{r}=-0.331, \mathrm{p}<.05)$ on select memory measures (consistent long term recall, long term storage, delayed recall). Other studies have shown that patients with stronger preoperative fMRI activation are at higher risk of greater postoperative memory decline $\mathrm{e}^{82}$ and those with greater ipsilateral compared with contralateral medial temporal lobe activation had greater memory decline following anterior temporal lobe resection $^{78}$.

Thus, memory fMRI can predict postoperative memory decline when comparing patients to controls. It is important to note that the aforementioned studies compared groups of patients to groups of control subjects. To be clinically relevant, however, memory fMRI needs to be predictive of post-operative outcome on a single-subject basis. Only when this is achieved can memory fMRI supplant the IAP. There are a number of reasons why single subject memory fMRI is not yet clinically viable. The most important reason is that a memory fMRI paradigm has not yet been developed that produces robust activation that can be reliably seen in single subjects ${ }^{81}$. Indeed, the inherent complexity of memory with different types of memory (verbal vs. nonverbal, semantic, episodic, visual, etc.) makes the development of fMRI paradigms challenging. As a result, normal functioning patients can show poor or minimal memory fMRI activation. Should this occur, other fMRI paradigms may be tried or alternative testing (e.g., neuropsychological or the IAP) needs to be considered. Currently employed memory fMRI paradigms include verbal encoding and retrieval tasks as well as comparisons of novel versus repeated stimuli ${ }^{77,78}$. Other problems relate to tissue-air interfaces and draining veins in the mesial temporal regions that interfere with the BOLD signal ${ }^{77}$. Furthermore, post-surgical memory fMRI activation changes have been seen without decreases in verbal memory function ${ }^{79}$. Thus, even though the clinical viability of predictive fMRI memory scans is near, much of the success thus far remains on the group level rather than the individual patient level.
The reproducibility of memory fMRI has been assessed, but not in the field of epilepsy. In one study, patients with Alzheimer disease were scanned on three different occasions over 12 weeks and the mean and extent of activation within both hippocampi was found not to vary significantly between scans ${ }^{83}$. In addition, whole brain test-retest reliability indices were in the moderate to substantial range, leading the authors to conclude that memory fMRI may be useful for longitudinal studies and early clinical trials in Alzheimer's disease. A study of ten healthy older adults and ten persons with mild cognitive impairment showed that the reproducibility of memory fMRI was similar between both groups ${ }^{84}$. Test-retest contrasts revealed few differences in the locations and level of activation, but there was a slight decrease in activity during the second scan for both groups, possibly due to familiarity with the paradigm. Lastly, a study of healthy controls using the same working memory fMRI paradigm at four different imaging centres showed that it is possible to obtain reproducible memory indices across different centres and scanners ${ }^{85}$. The average percent signal change was found to be the most reliable activity measure and the absolute number of activated voxels to be the least.

Given that the risk of memory and cognitive decline after temporal lobe resection is quite high, the establishment of effective tools to predict this decline pre-surgically is essential.

\section{MOTOR, SOMATOSENSORY, AND VISUAL fMRI}

Functional MRI assessment of language and memory is becoming more commonplace in large epilepsy centres since temporal lobe resection is the most common epilepsy surgical procedure. The role of fMRI is also expanding to include motor, somatosensory, and visual system mapping for extratemporal resections, though there are few published reports.

\section{Motor fMRI}

The motor cortex has been well studied by fMRI. Using paradigms involving finger tapping (at a constant frequency that can vary by centre), hand flexion-extension, sequential body part manipulation, complex hand movements and others, fMRI activation maps can be generated that closely match those obtained through cortical stimulation ${ }^{86-88}$. Motor fMRI has proven to be reproducible both at the single subject and group level ${ }^{89,90}$. Scan-rescan paradigms showed robust premotor, primary motor, and supplementary motor area activation within subjects over long periods of time (two months or greater) ${ }^{89}$ and within groups, even when performed at different sites with different scanners ${ }^{90}$. Though there are no specific guidelines for the exact rate at which motor tasks should be performed, a direct correlation has been found between tapping frequency and BOLD activation ${ }^{91}$. It is therefore advisable that subjects perform these tasks at a constant rate that will not introduce movement artefact into the data. The rate should also be constant between patients to ensure consistency and reliability of motor fMRI at a given centre. An example motor map from a hand coordination task during a clinical fMRI study performed in our centre is shown in Figure 4. Additionally, since motor tasks are relatively simple, motor fMRI can also gauge a patient's ability to perform more complex tasks during a scan such as language or memory tasks. However, this is not commonly done. 


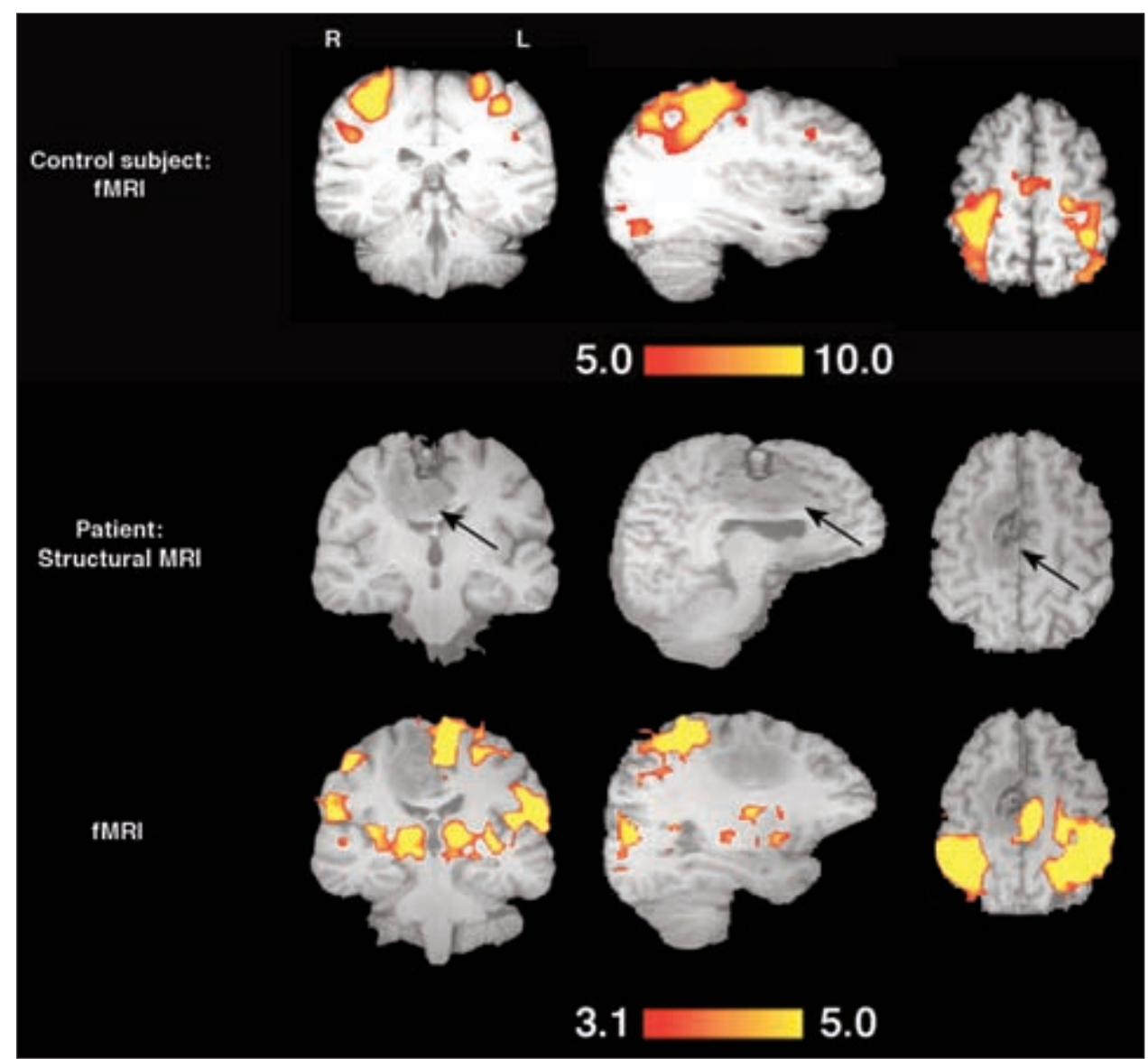

Figure 4: Motor fMRI. Brain activation during a simple bimanual motor task in a control subject and a patient with epilepsy secondary to a low-grade glioma. Top row: Brain activation in a healthy control subject, shown in coronal, saggital, and axial views. Middle and bottom rows: Structural and functional imaging of a patient showing a low grade glioma in the right frontal lobe (arrow). Of note, there is no significant motor activation within the glioma, suggesting that the lesion may be removed without threat of major motor deficit.

Functional MRI activation maps provide excellent localization for surgical decision-making in patients where resective surgery may border areas of motor function ${ }^{86,87,92}$. A study of patients with tumours near the central sulcus showed an $84 \%$ and $83 \%$ concordance between fMRI and cortical stimulation for motor and sensory cortices, respectively ${ }^{93}$. In a study of pre-surgical patients with epilepsy, sensorimotor fMRI was found to be a useful assessment tool in three key stages of pre-operative management: fMRI was used to determine the feasibility of proposed surgical resection margins, it aided in surgical planning, and it helped determine which patients would benefit most from invasive monitoring coverage ${ }^{87}$. Another study compared cortical stimulation to fMRI for localizing motor function in pre-operative epilepsy patients ${ }^{92}$. Using stimulation of the motor cortex as a reference, motor fMRI was found to have a sensitivity of 1.0 (responses which correctly identified motor area) and a specificity of 0.96 (correctly identified negative responses); however, the sample size was small (four subjects). In the pre-operative assessment of children with epilepsy, fMRI may also play an important role in surgical decision-making. Forty-three children with lesional focal epilepsy underwent motor fMRI as part of their pre-surgical assessment. The fMRI data provided useful information that contributed to the determination of whether the brain lesion was adequately distant from the motor cortex, and it helped establish which patients required invasive monitoring, and it revealed postoperative functional reorganization in $74 \%$ of children studied $^{86}$. The authors stressed the importance of motor fMRI assessment when considering any epilepsy surgery near the central sulcus.

Though the bulk of the clinical applications of fMRI is related to language and memory, the clinical role of motor, sensory, and visual fMRI is increasing. This lag may be, in part, due to the relative infrequency of frontal, parietal, and occipital resective surgeries compared to temporal lobe resections in patients with epilepsy. While cortical stimulation mapping of motor regions remains the definitive surgical guide, our epilepsy centre uses motor fMRI to help direct cortical stimulation. The 
fMRI activation maps help surgeons limit the breadth of the cortical stimulation, improving its overall accuracy and decreasing the time required.

\section{Somatosensory fMRI}

Clinical somatosensory fMRI has typically been used to assess sensory plasticity following limb amputation, skin grafts, or spinal cord injury. Applications in epilepsy surgery are limited to paediatric hemispherectomy. Two fMRI studies documented reorganization of somatosensory maps following hemispherectomies in children. One study of two subjects showed both sensory and motor function reallocation post hemispherectomy to the remaining hemisphere, although none of the redistributed activity was seen in the primary motor or sensory cortices ${ }^{94}$. The authors hypothesized that the combination of associative zones such as the posterior area of the somatosensory cortex, along with the primary and supplementary motor areas, may be sufficient for the perception of the sensory stimuli. The second published report detailed fMRI activation changes after two weeks of intense locomotor therapy following hemispherectomy in three children ${ }^{95}$. Increases in the spatial extent and intensity of voxel activation were found in all patients in the examined ROIs (including the primary sensorimotor and secondary somatosensory cortices). Healthy control subjects who underwent the same physical therapy and fMRI tests showed global decreases in these same regions. The authors suggested that this application for fMRI in epilepsy surgery may help monitor the effectiveness of postoperative physical therapy.

\section{Visual fMRI}

Visual fMRI may predict postoperative visual deficits for seizure foci in or adjacent to the occipital lobe ${ }^{96}$. Although no reports of this possibility have been published to date, one promising study showed that visual fMRI may help identify seizure foci in occipital lobe epilepsy ${ }^{97}$. An asymmetric activation map of the visual cortex activation was found to be an excellent predictor of the side of the seizure focus. Specifically, fMRI activation during full-field visual stimulation (black-white checkerboard) revealed abnormal activation patterns on the side of the seizure focus in all ten subjects studied. In contrast, only one of nine control subjects displayed potentially abnormal activation patterns of activation. Thus, while the results of preliminary studies are promising, visual fMRI is not used in routine clinical practice at the present time.

A novel technique for visual field assessment has been developed outside of the field of epilepsy using combined DTI$\mathrm{fMRI}^{98-100}$. Visual system recovery was assessed in two case studies: a 24-year-old female with ischemic injury to the right calcarine region ${ }^{98}$ and an infant with a left temporo-parietooccipital perinatal stroke ${ }^{100}$. In both cases, combined DTI-fMRI provided valuable data regarding the patient's progress poststroke; changes in the patients' fractional anisotropy and gross voxel activation during the recovery of visual processes after injury provided novel information about these types of lesions and can help predict disease evolution. The authors concluded that this technique may be useful for following patient recovery and guiding rehabilitation. Another study of 16 patients with visual field deficits caused by trauma, infarct, inflammation to the occipital lobe or optic nerve showed that combined DTI-
fMRI was useful in assessing the efficacy of hyperbaric oxygen treatment on these lesions ${ }^{99}$. DTI-fMRI provided quantitative evidence of patient improvement with hyperbaric oxygen therapy by demonstrating increases in fractional anisotropy and voxel activation after treatment. The authors concluded that DTI-fMRI was useful in evaluating patient response to hyperbaric oxygen treatment in these patients.

\section{EEG-fMRI}

Identifying the seizure focus is one of the most important steps in the pre-surgical assessment of an epilepsy surgery candidate, but it can also prove to be a challenging task. Traditional, minimally invasive localization techniques such as scalp EEG, structural MRI, PET and SPECT have proven successful in the majority of cases ${ }^{101,102}$. However, in spite of many advanced localization techniques, approximately $15 \%$ of surgical candidates may be rejected for surgery due to an inability to localize the seizure focus ${ }^{101}$. One tool that may aid in seizure localization is simultaneous EEG-fMRI.

Ives and colleges (1993) first demonstrated that EEG could be effectively and reliably collected during an MR scan with sufficient preparation and post-processing ${ }^{103}$. Since then, EEGfMRI has become a burgeoning and important research tool in the study of epilepsy. Numerous insights into the generation of interictal and ictal discharges have been made through the use of EEG-fMRI ${ }^{23,102,104-108}$. An example of simultaneous EEG-fMRI data collected in our centre is shown in Figure 5. The theoretical advantage of EEG-fMRI in epilepsy surgery relates to better identifying a potential seizure focus. Specifically, the correlation of an epileptiform discharge to a region of BOLD signal may provide a novel, three-dimensional visualization of a potential seizure focus. It is important to note that ictal and interictal foci may not always be at the same location. For example, previous EEG-fMRI studies have shown that patients with different types of malformations of cortical development may have interictal and ictal discharges associated with BOLD signal changes in different brain regions ${ }^{23}$.

Despite this limitation, EEG-fMRI provides reproducible results between studies ${ }^{109}$. By analyzing similar interictal discharges recorded from patients scanned on two separate occasions, maps of BOLD signal changes were congruent in 9/15 cases. Scans were performed at 1.5 and 3.0T, and higher field strengths increased the reproducibility of EEG-fMRI data. Also, a greater number of recorded discharges also increased reproducibility. Further discussion of the technical requirements, specifications, implementation, and limitations of EEG-fMRI can be found in a review published by our group previously ${ }^{110}$.

\section{Interictal EEG-fMRI}

The unpredictability of seizure onset makes capturing one in a finite time during an fMRI scan difficult. As such, the majority of published EEG-fMRI literature examines interictal discharges. One recent study evaluated the utility of EEG-fMRI in the pre-surgical workup for epilepsy patients ${ }^{102}$. In this study, 29 patients initially rejected for epilepsy surgery using traditional methods were re-evaluated using interictal EEGfMRI. These patients had been rejected for surgery because of an unclear focus, presumed multifocality, or a combination of both. The authors performed EEG-fMRI studies, looking for patients 


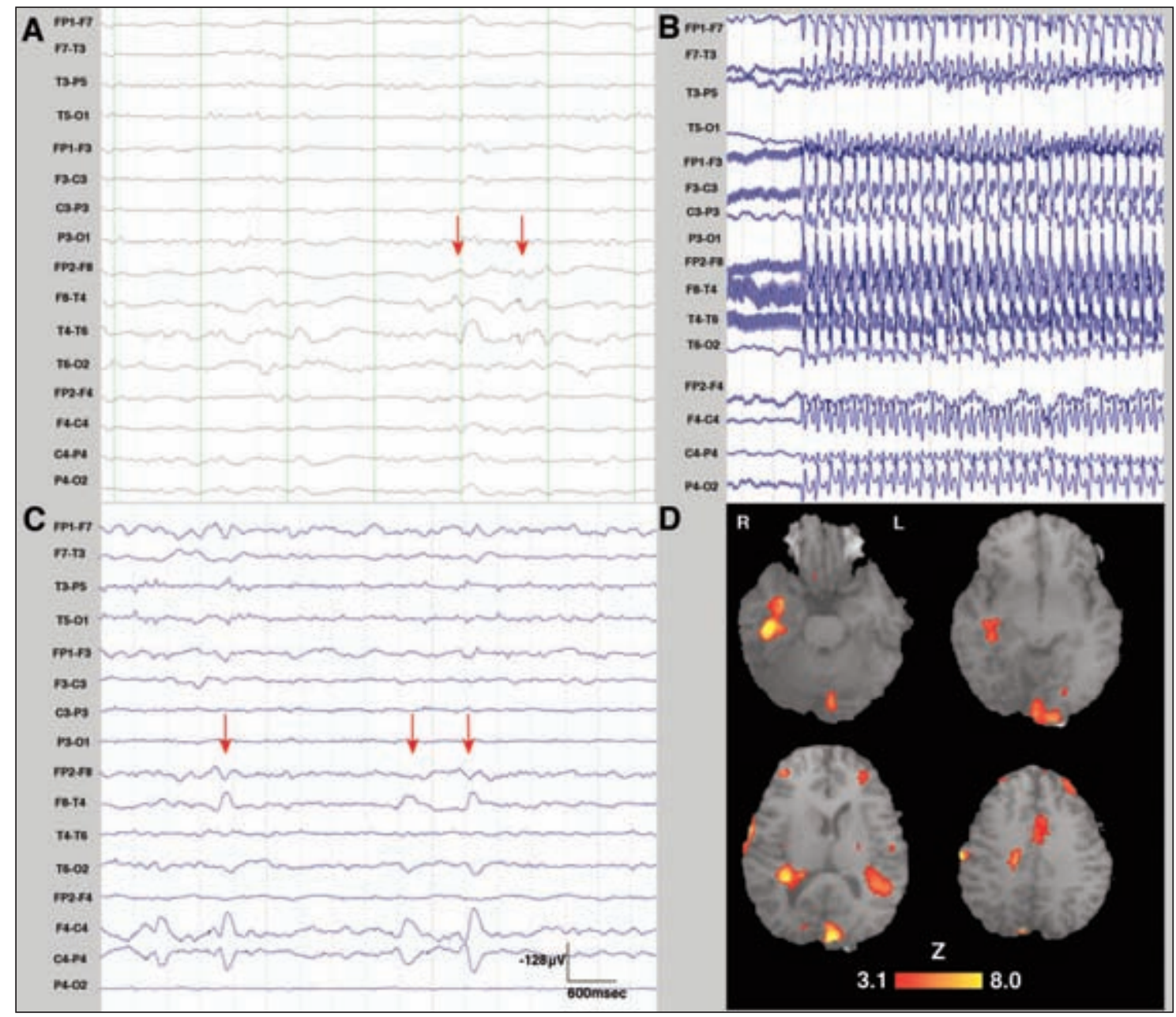

Figure 5: EEG-fMRI data collected at 3T. A. EEG data obtained under ideal conditions in hospital. B. Raw EEG data obtained during acquisition of fMRI images. C. Same data after post-processing to remove MR gradient switching artefact. Interictal discharges are identified by arrows. D. BOLD signals associated with the interictal discharges shown in C. The correlation of epileptiform discharges to BOLD signal may provide a novel, three-dimensional visualization of seizure foci.

in whom at least one BOLD response was significant and colocalized with the interictal discharges. Of the 29 patients, eight had positive BOLD responses that were topographically related to a suspected seizure focus. These eight patients were initially rejected for surgery because they had an unclear focus $(n=3)$, presumed multifocality $(n=2)$, or both $(n=3)$. The patients were re-evaluated using the EEG-fMRI data and improved localization was seen in four of the six patients with unclear foci and one of the five patients with presumed multifocality. Thus, EEG-fMRI provided clinically relevant information that improved seizure localization in these subjects. In two of these patients, intracranial VEM supported the EEG-fMRI data. In addition, EEG-fMRI also provided negative results that corroborated the clinical decision not to provide surgery to some patients.

Interictal EEG-fMRI also proved useful in the clinical assessment of patients with nonlesional frontal lobe epilepsy, which is a difficult group to assess pre-surgically ${ }^{111}$. These patients are often not considered for surgery or have poorer postoperative outcomes due to the difficulty in determining an epileptogenic focus. In this study, nine patients with non-lesional frontal lobe epilepsy were assessed using interictal EEG-fMRI and their maps of BOLD activation were compared to previous assessments including PET and SPECT, structural MR, and postoperative outcomes. The areas of peak BOLD signal changes matched PET and SPECT results very well; the maximum BOLD signal corresponded to reduced glucose metabolism in $100 \%$ of the PET studies (3/3) and hyperperfusion seen on SPECT matched the BOLD response in $75 \%$ of the patients (3/4). EEG-fMRI results also identified potentially pathologic sulci not previously noted on structural MR in two patients, and maximum BOLD activation was seen within the resected margin of another patient who has been seizure-free for two years following resection. The authors suggest that EEG-fMRI should be used in some circumstances in this challenging group of patients. 


\section{Ictal EEG-fMRI}

Capturing ictal events during an EEG-fMRI scanning session is challenging due to the unpredictable nature of their occurrence. Despite this, several ictal EEG-fMRI studies have been published ${ }^{22,112,113}$. Of particular note, EEG-fMRI proved to valuable for identifying the ictal epileptogenic zone when subclinical seizures were detected ${ }^{22}$. Indeed, these subclinical ictal events were ideal for EEG-fMRI analysis as they lacked significant clinical manifestations that would contaminate fMRI data with motion artefact. Ictal EEG-fMRI studies continue to be performed and they may provide further insight into seizure generation and propagation, which in turn, will further guide surgical decision-making.

\section{EEG-fMRI and Post Operative Outcomes}

It is essential to correlate EEG-fMRI data with postsurgical outcomes in order to validate EEG-fMRI as a valid pre-operative assessment tool. However, few data have been published in this regard. One report of ten post-surgical patients studied with interictal EEG-fMRI found seven of the ten patients to be seizure free post-operatively ${ }^{114}$. Of those seven patients, six showed strong concordance between the location of maximum BOLD signal increases and tissue resection. These preliminary data suggest that EEG-fMRI may have a valuable role in pre-surgical assessments. Further research is required to establish validity across different patient sub-types.

\section{Advantages AND Disadvantages OF fMRI}

Functional MRI has a number of advantages over some of the conventional investigations currently used in the pre-surgical workup of epilepsy patients. Firstly, fMRI is decidedly noninvasive. Compared to IAP or cortical stimulation, fMRI requires no injection of medication, no surgical procedure, and carries virtually no risk of complication. This allows the procedure to be performed in a wider population of patients, including children ${ }^{50}$. Also, unlike other forms of structural and functional neuroimaging such as CT, SPECT and PET, there is no exposure to ionizing radiation. Because fMRI is non-invasive and carries no risk of radiation exposure, studies are easily repeatable. Functional MRI scans can also be performed widely as most MR scanners are equipped with the appropriate hardware to acquire functional data. Acquiring fMRI data requires no specialized clinical personnel apart from an experienced MRI technologist and can be performed in less than one hour in both an inpatient and outpatient setting.

There remain limitations and disadvantages to fMRI scanning. The spatial resolution of fMRI data is lower compared to a typical T1-weighted anatomical MR $\operatorname{scan}^{50}$. The relatively low signal strength compared to background noise for the BOLD signal is noted as one of the causes. Additionally, the BOLD signal itself is not yet entirely understood: the relationship between positive and negative BOLD signals and alterations in neural activity has not been fully established ${ }^{115}$. Lastly, although acquiring fMRI data is relatively uncomplicated in centres equipped with MR scanners, developing an fMRI program requires specialized personnel such as fMRI physicists to develop, test, and implement fMRI paradigms. Furthermore, once acquired, fMRI data also requires specially trained personnel to analyze the data and generate maps of activation. Current MR scanner manufacturers are developing user-friendly image analysis programs that will allow MR technologists to perform the analysis themselves, however, they are not sufficiently robust for routine use at the present time.

EEG-fMRI has a few limitations as well. It requires subjects to have frequent epileptiform discharges (optimally $>1$ discharge per minute, but fewer may not preclude a study) that can be recorded and studied in the MR scanner. Hence, relatively few patients would meet this criterion. In addition, the precise spatial and temporal relationship between interictal discharges and the BOLD response is not completely understood.

\section{Novel DeVELOPMENTS}

The field of fMRI in epilepsy is rapidly expanding due to the proliferation of clinically- and research-based fMRI studies. Of these, three new fields of research may eventually have an impact on the pre-operative assessment of patients with epilepsy: functional connectivity, the pre-ictal state, and combined intracranial EEG-fMRI.

\section{Connectivity}

Functional connectivity can be conceptualized as the temporal correlation between neural activity in anatomically distinct brain regions. By examining the temporal correlations of BOLD signal fluctuations between different regions, fMRI can measure functional connectivity and help study cortical reorganization in the epileptic brain or identify potential epileptogenic networks. Task-related connectivity measures BOLD signal fluctuations during specific tasks, whereas resting state connectivity measures the degree of synchrony of low frequency $(<0.1 \mathrm{~Hz})$ BOLD signal fluctuations across the brain at rest ${ }^{116}$. Although the exact relationship between BOLD signal and neural activity at rest has not yet been delineated, resting state connectivity has nonetheless been applied to patients with temporal lobe epilepsy ${ }^{58,117-119}$. To this end, subjects with left temporal lobe epilepsy showed significantly less functional connectivity between language areas compared to control subjects, which may underlie the language difficulties these patients experience ${ }^{58}$. Another study demonstrated a loss of cross-hemispheric connectivity between hippocampi in patients with unilateral hippocampal sclerosis compared to controls ${ }^{118}$. Unilateral hippocampal network connectivity loss has also been found ipsilateral to the seizure focus and connectivity within the contralateral hippocampal network increased to potentially compensate for this ${ }^{119,120}$. In the future, resting state connectivity analysis could therefore be useful to assess the language or memory system in patients who are unable to perform traditional language or memory tasks.

EEG-fMRI data may also be used to study functional connectivity in epileptogenic networks ${ }^{121,122}$. Two techniques for EEG-fMRI connectivity analysis have thus far been published. The first involves the identification of regions showing spikeassociated BOLD signal increases and using them as seed regions for functional connectivity analyses ${ }^{121}$. Alternatively, EEG data itself can be used to determine the periods during which epileptiform discharges are seen and comparing connectivity during these times to periods during which no discharges are seen on $\mathrm{EEG}^{122}$. 
Published data examining idiopathic generalized epilepsy showed spike-associated BOLD signal changes in the thalamus, precuneus, frontomesial cortex, and cerebellum. These regions were then used as seeds for connectivity analysis ${ }^{121}$. However, the connectivity between these regions and the rest of the brain were not significantly different between patients and controls. Resting state functional connectivity using EEG-fMRI was also used to investigate interhemispheric connections in childhood absence epilepsy ${ }^{122}$. Comparing resting functional connectivity between CAE patients and healthy controls, increased hemispheric connectivity was found in many seizure related regions. These changes were found during seizures, but also when no seizure activity was detected ${ }^{122}$. The authors compared resting state functional connectivity between patients and healthy controls in 16 pairs of seizure-related regions of interest $^{122}$. Increased connectivity was found between hemispheres in the lateral orbitofrontal cortex in patients compared to control subjects even when seizures were not occurring. This suggests that the lateral orbitofrontal cortex may play a significant role in the ictal or interictal manifestations of childhood absence epilepsy ${ }^{122}$.

Default mode network connectivity is another area of interest in epilepsy. The default mode network is a group of structures that demonstrate activation during resting wakefulness that is temporarily suspended during tasks requiring acute attention ${ }^{123}$. These structures include, but are not limited to the medial frontal cortex, precuneus and posterior cingulate. Connectivity between these structures can easily be assessed in the resting-state. To this end, the resting state connectivity of the default mode network connectivity has been shown to be altered in a variety of neurological disorders including epilepsy ${ }^{124}$. Two recent studies have investigated the default mode network in patients with epilepsy ${ }^{125,126}$. One study compared default mode network connectivity between controls and patients with complex partial seizures during rest periods between language tasks ${ }^{125}$. Patients had more widespread default network activity during rest following the language tasks, which suggests an impairment in switching from task-related activities to rest in patients with epilepsy. Another recent study compared resting state connectivity between patients with absence epilepsy and controls ${ }^{126}$. Decreased default mode network connectivity was seen in patients, even when no interictal discharges were detected. In addition, the connectivity in some of regions was negatively correlated with the duration of epilepsy. The authors suggested that absence epilepsy is associated with abnormal functional integration in default mode network that may underlie the cognitive changes seen during absence seizures.

At the present time, the aforementioned studies of functional connectivity are providing mechanistic insights into the pathophysiology of focal and generalized epilepsy. In the future, similar studies may provide meaningful input into surgical decision making, perhaps by identifying an ictal focus or by helping to stratify the risk of cognitive deficits following resective surgery.

\section{Pre-ictal State}

The mechanisms underlying the transition into seizures are not well understood ${ }^{127}$. Converging evidence supports the presence of a "pre-ictal" period during which seizures evolve over minutes to hours. Increasing, but incomplete, evidence suggests that alterations in specific biomarkers may be predictive of seizure onset. These markers include changes in neuronal phase relations between the seizure focus and surrounding regions, increased blood flow, and increased neuronal synchronization. These physiological processes will generate BOLD signals and therefore fMRI may prove to be an important tool for studying the pre-ictal state and identifying seizure foci. To this end, we have shown that brain activation changes occur up to 20 minutes prior to seizure onset at the seizure focus and elsewhere in the brain ${ }^{128}$. We are currently expanding on these preliminary findings. Characterizing the spatiotemporal patterns of pre-ictal brain activation will further our understanding of the transition into seizures. It may also provide information about the timing and location of critical preictal physiological processes. This information, in turn, may be used to identify targets for novel therapeutic interventions in the future (e.g., deep-brain stimulators, direct drug delivery devices, etc.).

\section{Intracranial EEG-fMRI}

Scalp EEG-fMRI studies have provided a number of insights in the study of epilepsy. However, scalp EEG is limited to detecting discharges involving a large cortical surface area $(>10$ $\left.\mathrm{cm}^{2}\right)^{129}$. Intracranial EEG electrodes circumvent this limitation through their ability to detect low amplitude, deeply originating discharges. Thus combining intracranial EEG with fMRI would greatly increase the sensitivity of EEG-fMRI, and it may yield increasingly accurate seizure focus localization. We first reported our intracranial EEG-fMRI data at $3.0 \mathrm{~T}^{130}$ and this was subsequently followed by another group at $1.5 \mathrm{~T}^{131}$. It is likely that these data will yield new insights into the generation of interictal discharges, given the unprecedented sensitivity with which EEG-fMRI can now be performed.

\section{Conclusion}

During the pre-operative assessment of patients with epilepsy, a variety of imaging and clinical investigations are used to confirm a diagnosis of epilepsy, localize epileptogenic foci, and predict surgical outcome. Functional MRI is a comparatively new technique that is increasingly being utilized for these preoperative assessments. Functional MRI techniques such as language fMRI are used routinely in many epilepsy centres. Other fMRI techniques such as motor, memory, and EEG-fMRI studies are moving towards routine clinical implementation, but further refinements are necessary. Advanced fMRI techniques such as intracranial EEG-fMRI or connectivity analysis will likely provide key insights into epileptogenesis that may eventually directly benefit patients suffering from epilepsy.

\section{ACKNOWLEDGEMENTS}

The authors thank E.G. Slone and I. Gaxiola-Valdez for critically reviewing the manuscript. Dr. Federico is funded by the Canadian Institutes of Health Research.

\section{REFERENCES}

1. Noachtar S, Borggraefe I. Epilepsy surgery: a critical review. Epilepsy Behav. 2009;15(1):66-72. 
2. French JA. Refractory epilepsy: clinical overview. Epilepsia. 2007; 48 Suppl 1:3-7.

3. Spencer S, Huh L. Outcomes of epilepsy surgery in adults and children. Lancet Neurol. 2008;7(6):525-37.

4. Foldvary N, Bingaman WE, Wyllie E. Surgical treatment of epilepsy. Neurol Clin. 2001;19(2):491-515.

5. Placantonakis DG, Schwartz TH. Localization in epilepsy. Neurol Clin. 2009;27(4):1015-30.

6. Cascino GD. Video-EEG monitoring in adults. Epilepsia. 2002;43 Suppl 3:80-93

7. Schauble B, Cascino GD. Advances in neuroimaging: management of partial epileptic syndromes. Neurosurg Rev. 2003;26(4):23346; discussion 247-8.

8. Duncan JS. Imaging and epilepsy. Brain. 1997;120 ( Pt 2):339-77.

9. Maehara T. Neuroimaging of epilepsy. Neuropathology. 2007;27(6):585-93.

10. Matsuda H, Matsuda K, Nakamura F, et al. Contribution of subtraction ictal SPECT coregistered to MRI to epilepsy surgery: a multicenter study. Ann Nucl Med. 2009;23(3):283-91.

11. Richardson M. Current themes in neuroimaging of epilepsy: brain networks, dynamic phenomena, and clinical relevance. Clin Neurophysiol. 2010;121(8):1153-75.

12. Richardson MP. Epilepsy and surgical mapping. Br Med Bull. 2003:65:179-92.

13. Lee BI, Markand ON, Siddiqui AR, et al. Single photon emission computed tomography (SPECT) brain imaging using N,N,N'trimethyl-N'-(2 hydroxy-3-methyl-5-123I-iodobenzyl)-1,3propanediamine $2 \mathrm{HCl}$ (HIPDM): intractable complex partial seizures. Neurology. 1986;36(11):1471-7.

14. Launes J, Iivanainen M, Salmi T, Nikkinen $P$, Lindroth L, Liewendahl K. Interictal brain 99Tc-HM-PAO SPECT hypoperfusion in patients with unstable partial epilepsy and normal CT. Acta Neurol Scand. 1992;86(6):558-62.

15. Ryvlin P, Garcia-Larrea L, Philippon B, et al. High signal intensity on T2-weighted MRI correlates with hypoperfusion in temporal lobe epilepsy. Epilepsia. 1992;33(1):28-35.

16. Knowlton RC. The role of FDG-PET, ictal SPECT, and MEG in the epilepsy surgery evaluation. Epilepsy Behav. 2006;8(1):91-101.

17. Ray A, Bowyer SM. Clinical applications of magnetoencephalography in epilepsy. Ann Indian Acad Neurol. 2010;13 (1):14-22.

18. Stufflebeam SM, Tanaka N, Ahlfors SP. Clinical applications of magnetoencephalography. Hum Brain Mapp. 2009;30(6): 1813-23.

19. Stufflebeam SM. Clinical magnetoencephalography for neurosurgery. Neurosurg Clin N Am. 2011;22(2):153-67, vii-viii

20. Najib U, Bashir S, Edwards D, Rotenberg A, Pascual-Leone A. Transcranial brain stimulation: clinical applications and future directions. Neurosurg Clin N Am. 2011;22(2):233-51, ix.

21. Groening K, Brodbeck V, Moeller F, et al. Combination of EEGfMRI and EEG source analysis improves interpretation of spikeassociated activation networks in paediatric pharmacoresistant focal epilepsies. Neuroimage. 2009;46(3):827-33.

22. LeVan P, Tyvaert L, Moeller F, Gotman J. Independent component analysis reveals dynamic ictal BOLD responses in EEG-fMRI data from focal epilepsy patients. Neuroimage. 2010;49(1): 366-78

23. Tyvaert L, Hawco C, Kobayashi E, LeVan P, Dubeau F, Gotman J. Different structures involved during ictal and interictal epileptic activity in malformations of cortical development: an EEG-fMRI study. Brain. 2008;131(Pt 8):2042-60

24. Siegel AM. Presurgical evaluation and surgical treatment of medically refractory epilepsy. Neurosurg Rev. 2004;27(1):1-18; discussion 19-21.

25. Curatolo JM, Macdonell RA, Berkovic SF, Fabinyi GC. Intraoperative monitoring to preserve central visual fields during occipital corticectomy for epilepsy. J Clin Neurosci. 2000;7(3): 234-7.

26. Ogawa S, Lee TM, Nayak AS, Glynn P. Oxygenation-sensitive contrast in magnetic resonance image of rodent brain at high magnetic fields. Magn Reson Med. 1990;14(1):68-78.
27. Benar CG, Gross DW, Wang Y, et al. The BOLD response to interictal epileptiform discharges. Neuroimage. 2002;17(3): 1182-92.

28. Keogh BP, Cordes D. Quantitative approaches to functional MRI: applications in epilepsy. Epilepsia. 2007;48 Suppl 4:27-36.

29. Sullivan JE, 3rd, Detre JA. Functional magnetic resonance imaging in the treatment of epilepsy. Curr Neurol Neurosci Rep. 2005;5 (4):299-306.

30. Logothetis NK. The underpinnings of the BOLD functional magnetic resonance imaging signal. J Neurosci. 2003;23(10): 3963-71.

31. Menon RS. Imaging function in the working brain with fMRI. Curr Opin Neurobiol. 2001;11(5):630-6.

32. Malonek D, Grinvald A. Interactions between electrical activity and cortical microcirculation revealed by imaging spectroscopy: implications for functional brain mapping. Science. 1996;272 (5261):551-4

33. Haller S, Bartsch AJ. Pitfalls in FMRI. Eur Radiol. 2009;19(11): 2689-706.

34. Bookheimer S. Pre-surgical language mapping with functional magnetic resonance imaging. Neuropsychol Rev. 2007;17(2): $145-55$.

35. Swanson SJ, Sabsevitz DS, Hammeke TA, Binder JR. Functional magnetic resonance imaging of language in epilepsy. Neuropsychol Rev. 2007;17(4):491-504.

36. Aldenkamp AP, Boon PA, Deblaere K, et al. Usefulness of language and memory testing during intracarotid amobarbital testing: observations from an fMRI study. Acta Neurol Scand. 2003; 108(3):147-52.

37. Arora J, Pugh K, Westerveld M, Spencer S, Spencer DD, Todd Constable R. Language lateralization in epilepsy patients: fMRI validated with the Wada procedure. Epilepsia. 2009;50(10): 2225-41.

38. Binder JR, Swanson SJ, Sabsevitz DS, Hammeke TA, Raghavan M, Mueller WM. A comparison of two fMRI methods for predicting verbal memory decline after left temporal lobectomy: language lateralization versus hippocampal activation asymmetry. Epilepsia. 2010;51(4):618-26.

39. Szaflarski JP, Holland SK, Jacola LM, Lindsell C, Privitera MD, Szaflarski M. Comprehensive presurgical functional MRI language evaluation in adult patients with epilepsy. Epilepsy Behav. 2008;12(1):74-83.

40. Fernandes MA, Smith ML, Logan W, Crawley A, McAndrews MP. Comparing language lateralization determined by dichotic listening and fMRI activation in frontal and temporal lobes in children with epilepsy. Brain Lang. 2006;96(1):106-14.

41. Pelletier I, Sauerwein HC, Lepore F, Saint-Amour D, Lassonde M. Non-invasive alternatives to the Wada test in the presurgical evaluation of language and memory functions in epilepsy patients. Epileptic Disord. 2007;9(2):111-26.

42. Wada J, Rasmussen T. Intracarotid injection of sodium amytal for the lateralization of cerebral speech dominance. J Neurosurg. 1960;106(6):1117-33

43. Loddenkemper T, Morris HH, Moddel G. Complications during the Wada test. Epilepsy Behav. 2008;13(3):551-3.

44. Jones-Gotman M, Sziklas V, Djordjevic J, et al. Etomidate speech and memory test (eSAM): a new drug and improved intracarotid procedure. Neurology. 2005;65(11):1723-9.

45. Jones-Gotman M, Sziklas V, Djordjevic J. Intracarotid amobarbital procedure and etomidate speech and memory test. Can J Neurol Sci. 2009;36 Suppl 2:S51-4.

46. Gallentine WB, Mikati MA. Intraoperative electrocorticography and cortical stimulation in children. J Clin Neurophysiol. 2009; 26(2):95-108

47. Hamberger MJ, Seidel WT, McKhann GM 2nd, Perrine K, Goodman RR. Brain stimulation reveals critical auditory naming cortex. Brain. 2005;128(Pt 11):2742-9.

48. Hartman AL, Lesser RP. Update on epilepsy and cerebral localization. Curr Neurol Neurosci Rep. 2007;7(6):498-507.

49. Ojemann G, Ojemann J, Lettich E, Berger M. Cortical language localization in left, dominant hemisphere. An electrical stimulation mapping investigation in 117 patients. J Neurosurg. 1989;71(3):316-26. 
50. Chakraborty A, McEvoy AW. Presurgical functional mapping with functional MRI. Curr Opin Neurol. 2008;21(4):446-51.

51. Powell HW, Duncan JS. Functional magnetic resonance imaging for assessment of language and memory in clinical practice. Curr Opin Neurol. 2005;18(2):161-6.

52. Binder JR, Sabsevitz DS, Swanson SJ, Hammeke TA, Raghavan M, Mueller WM. Use of preoperative functional MRI to predict verbal memory decline after temporal lobe epilepsy surgery. Epilepsia. 2008;49(8):1377-94.

53. Binder JR. Preoperative prediction of verbal episodic memory outcome using FMRI. Neurosurg Clin N Am. 2011;22(2):21932 , ix.

54. Binder JR, Gross WL, Allendorfer JB, et al. Mapping anterior temporal lobe language areas with fMRI: a multicenter normative study. Neuroimage. 2011;54(2):1465-75.

55. Binder JR. Functional MRI is a valid noninvasive alternative to Wada testing. Epilepsy Behav. 2011;20(2):214-22.

56. Engstrom M, Ragnehed M, Lundberg P, Soderfeldt B. Paradigm design of sensory-motor and language tests in clinical fMRI. Neurophysiol Clin. 2004;34(6):267-77.

57. Gaillard WD, Berl MM, Duke ES, et al. fMRI language dominance and FDG-PET hypometabolism. Neurology. 2011;76(15): $1322-9$.

58. Waites AB, Briellmann RS, Saling MM, Abbott DF, Jackson GD. Functional connectivity networks are disrupted in left temporal lobe epilepsy. Ann Neurol. 2006;59(2):335-43.

59. Desmond JE, Sum JM, Wagner AD, et al. Functional MRI measurement of language lateralization in Wada-tested patients. Brain. 1995;118 ( Pt 6):1411-19.

60. Rutten GJ, Ramsey NF, van Rijen PC, Alpherts WC, van Veelen CW. FMRI-determined language lateralization in patients with unilateral or mixed language dominance according to the Wada test. Neuroimage. 2002;17(1):447-60.

61. Giussani C, Roux FE, Ojemann J, Sganzerla EP, Pirillo D, Papagno C. Is preoperative functional magnetic resonance imaging reliable for language areas mapping in brain tumor surgery? Review of language functional magnetic resonance imaging and direct cortical stimulation correlation studies. Neurosurgery. 2010;66(1):113-20.

62. Rutten GJ, Ramsey NF, van Rijen PC, van Veelen CW. Reproducibility of fMRI-determined language lateralization in individual subjects. Brain Lang. 2002;80(3):421-37.

63. Fernandez G, Specht K, Weis S, et al. Intrasubject reproducibility of presurgical language lateralization and mapping using fMRI. Neurology. 2003;60(6):969-75.

64. Fedorenko E, Hsieh PJ, Nieto-Castanon A, Whitfield-Gabrieli S, Kanwisher N. New method for fMRI investigations of language: defining ROIs functionally in individual subjects. J Neurophysiol. 2010;104(2):1177-94

65. Gartus A, Foki T, Geissler A, Beisteiner R. Improvement of clinical language localization with an overt semantic and syntactic language functional MR imaging paradigm. AJNR Am J Neuroradiol. 2009;30(10):1977-85.

66. Fesl G, Bruhns P, Rau S, et al. Sensitivity and reliability of language laterality assessment with a free reversed association task--a fMRI study. Eur Radiol. 2010;20(3):683-95.

67. Rodrigo S, Oppenheim C, Chassoux F, et al. Language lateralization in temporal lobe epilepsy using functional MRI and probabilistic tractography. Epilepsia. 2008;49(8):1367-76.

68. Tie Y, Suarez RO, Whalen S, Radmanesh A, Norton IH, Golby AJ. Comparison of blocked and event-related fMRI designs for presurgical language mapping. Neuroimage. 2009;47 Suppl 2: T107-15.

69. Rutten GJ, Ramsey NF, van Rijen PC, Noordmans HJ, van Veelen $\mathrm{CW}$. Development of a functional magnetic resonance imaging protocol for intraoperative localization of critical temporoparietal language areas. Ann Neurol. 2002;51(3): 350-60.

70. Vigneau M, Beaucousin V, Herve PY, et al. Meta-analyzing left hemisphere language areas: phonology, semantics, and sentence processing. Neuroimage. 2006;30(4):1414-32.

71. Ellis TL, Stevens A. Deep brain stimulation for medically refractory epilepsy. Neurosurg Focus. 2008;25(3):E11.
72. Grattan-Smith JD, Harvey AS, Desmond PM, Chow CW. Hippocampal sclerosis in children with intractable temporal lobe epilepsy: detection with MR imaging. AJR Am J Roentgenol. 1993;161(5):1045-8

73. Nakatsuka T, Chen HX, Roper SN, Gu JG. Cannabinoid receptor-1 activation suppresses inhibitory synaptic activity in human dentate gyrus. Neuropharmacology. 2003;45(1):116-21 .

74. Wiebe S, Blume WT, Girvin JP, Eliasziw M. A randomized, controlled trial of surgery for temporal-lobe epilepsy. N Engl J Med. 2001;345(5):311-18.

75. Janszky J, Jokeit H, Kontopoulou K, et al. Functional MRI predicts memory performance after right mesiotemporal epilepsy surgery. Epilepsia. 2005;46(2):244-50.

76. Mechanic-Hamilton D, Korczykowski M, Yushkevich PA, et al. Hippocampal volumetry and functional MRI of memory in temporal lobe epilepsy. Epilepsy Behav. 2009;16(1):128-38.

77. Richardson MP, Strange BA, Duncan JS, Dolan RJ. Memory fMRI in left hippocampal sclerosis: optimizing the approach to predicting postsurgical memory. Neurology. 2006;66(5): 699-705.

78. Powell HW, Richardson MP, Symms MR, et al. Preoperative fMRI predicts memory decline following anterior temporal lobe resection. J Neurol Neurosurg Psychiatry. 2008;79(6):686-93.

79. Cheung MC, Chan AS, Lam JM, Chan YL. Pre- and postoperative fMRI and clinical memory performance in temporal lobe epilepsy. J Neurol Neurosurg Psychiatry. 2009;80(10):1099-106.

80. Trenerry MR, Jack CR, Jr., Ivnik RJ, et al. MRI hippocampal volumes and memory function before and after temporal lobectomy. Neurology. 1993;43(9):1800-5.

81. Bonelli SB, Powell RH, Yogarajah M, et al. Imaging memory in temporal lobe epilepsy: predicting the effects of temporal lobe resection. Brain. 2010;133(Pt 4):1186-99.

82. Labudda K, Mertens M, Aengenendt J, Ebner A, Woermann FG. Presurgical language fMRI activation correlates with postsurgical verbal memory decline in left-sided temporal lobe epilepsy. Epilepsy Res. 2010;92(2-3):258-61.

83. Atri A, O'Brien JL, Sreenivasan A, et al. Test-retest reliability of memory task functional magnetic resonance imaging in Alzheimer disease clinical trials. Arch Neurol. 2011;68(5): 599-606.

84. Clement F, Belleville S. Test-retest reliability of fMRI verbal episodic memory paradigms in healthy older adults and in persons with mild cognitive impairment. Hum Brain Mapp. 2009;30(12):4033-47.

85. Yendiki A, Greve DN, Wallace S, et al. Multi-site characterization of an fMRI working memory paradigm: reliability of activation indices. Neuroimage. 2010;53(1):119-31.

86. De Tiege X, Connelly A, Liegeois F, et al. Influence of motor functional magnetic resonance imaging on the surgical management of children and adolescents with symptomatic focal epilepsy. Neurosurgery. 2009;64(5):856-64; discussion 864.

87. Lee CC, Ward HA, Sharbrough FW, et al. Assessment of functional MR imaging in neurosurgical planning. AJNR Am J Neuroradiol. 1999;20(8):1511-19.

88. Meier JD, Aflalo TN, Kastner S, Graziano MS. Complex organization of human primary motor cortex: a high-resolution fMRI study. J Neurophysiol. 2008;100(4):1800-12.

89. Yoo SS, Wei X, Dickey CC, Guttmann CR, Panych LP. Long-term reproducibility analysis of fMRI using hand motor task. Int $\mathrm{J}$ Neurosci. 2005;115(1):55-77.

90. Gountouna VE, Job DE, McIntosh AM, et al. Functional Magnetic Resonance Imaging (fMRI) reproducibility and variance components across visits and scanning sites with a finger tapping task. Neuroimage. 2010;49(1):552-60.

91. Rao SM, Bandettini PA, Binder JR, et al. Relationship between finger movement rate and functional magnetic resonance signal change in human primary motor cortex. J Cereb Blood Flow Metab. 1996;16(6):1250-4

92. Mehta AD, Klein G. Clinical utility of functional magnetic resonance imaging for brain mapping in epilepsy surgery. Epilepsy Res. 2010;89(1):126-32. 
93. Majos A, Tybor K, Stefanczyk L, Goraj B. Cortical mapping by functional magnetic resonance imaging in patients with brain tumors. Eur Radiol. 2005;15(6):1148-58.

94. Graveline CJ, Mikulis DJ, Crawley AP, Hwang PA. Regionalized sensorimotor plasticity after hemispherectomy fMRI evaluation. Pediatr Neurol. 1998;19(5):337-42.

95. de Bode S, Mathern GW, Bookheimer S, Dobkin B. Locomotor training remodels fMRI sensorimotor cortical activations in children after cerebral hemispherectomy. Neurorehabil Neural Repair. 2007;21(6):497-508.

96. McFadzean RM, Condon BC, Barr DB. Functional magnetic resonance imaging in the visual system. J Neuroophthalmol. 1999;19(3):186-200.

97. Masuoka LK, Anderson AW, Gore JC, McCarthy G, Spencer DD, Novotny EJ. Functional magnetic resonance imaging identifies abnormal visual cortical function in patients with occipital lobe epilepsy. Epilepsia. 1999;40(9):1248-53.

98. Polonara G, Salvolini S, Fabri M, et al. Unilateral visual loss due to ischaemic injury in the right calcarine region: a functional magnetic resonance imaging and diffusion tension imaging follow-up study. Int Ophthalmol. 2011;31(2):129-34.

99. Chen Z, Ni P, Lin Y, et al. Visual pathway lesion and its development during hyperbaric oxygen treatment: a bold- fMRI and DTI study. J Magn Reson Imaging. 2010;31(5):1054-60.

100. Seghier ML, Lazeyras F, Zimine S, Saudan-Frei S, Safran AB, Huppi PS. Visual recovery after perinatal stroke evidenced by functional and diffusion MRI: case report. BMC Neurol. 2005; 5:17.

101.Berg AT, Vickrey BG, Langfitt JT, et al. The multicenter study of epilepsy surgery: recruitment and selection for surgery. Epilepsia. 2003;44(11):1425-33.

102.Zijlmans M, Huiskamp G, Hersevoort M, Seppenwoolde JH, van Huffelen AC, Leijten FS. EEG-fMRI in the preoperative workup for epilepsy surgery. Brain. 2007;130(Pt 9):2343-53.

103. Ives JR, Warach S, Schmitt F, Edelman RR, Schomer DL. Monitoring the patient's EEG during echo planar MRI. Electroencephalogr Clin Neurophysiol. 1993;87(6):417-20.

104. Bagshaw AP, Aghakhani Y, Benar CG, et al. EEG-fMRI of focal epileptic spikes: analysis with multiple haemodynamic functions and comparison with gadolinium-enhanced MR angiograms. Hum Brain Mapp. 2004;22(3):179-92.

105. Benar CG, Grova C, Kobayashi E, et al. EEG-fMRI of epileptic spikes: concordance with EEG source localization and intracranial EEG. Neuroimage. 2006;30(4):1161-70.

106. Federico P, Archer JS, Abbott DF, Jackson GD. Cortical/subcortical BOLD changes associated with epileptic discharges: an EEGfMRI study at 3 T. Neurology. 2005;64(7):1125-30.

107. Gotman J. Epileptic networks studied with EEG-fMRI. Epilepsia. 2008;49 Suppl 3:42-51

108. Vulliemoz S, Lemieux L, Daunizeau J, Michel CM, Duncan JS. The combination of EEG source imaging and EEG-correlated functional MRI to map epileptic networks. Epilepsia. 2010;51 (4):491-505.

109. Gholipour T, Moeller F, Pittau F, Dubeau F, Gotman J. Reproducibility of interictal EEG-fMRI results in patients with epilepsy. Epilepsia. 2011;52(3):433-42.

110. Cunningham CJ, Zaamout Mel F, Goodyear B, Federico P. Simultaneous EEG-fMRI in human epilepsy. Can J Neurol Sci. 2008;35(4):420-35.

111. Moeller F, Tyvaert L, Nguyen DK, et al. EEG-fMRI: adding to standard evaluations of patients with nonlesional frontal lobe epilepsy. Neurology. 2009;73(23):2023-30.

112.Li Q, Luo C, Yang T, et al. EEG-fMRI study on the interictal and ictal generalized spike-wave discharges in patients with childhood absence epilepsy. Epilepsy Res. 2009;87(2-3):160-8.
113. Liu Y, Yang T, Liao W, et al. EEG-fMRI study of the ictal and interictal epileptic activity in patients with eyelid myoclonia with absences. Epilepsia. 2008;49(12):2078-86

114. Thornton R, Laufs H, Rodionov R, et al. EEG correlated functional MRI and postoperative outcome in focal epilepsy. J Neurol Neurosurg Psychiatry. 2010;81(8):922-7.

115. Shmuel A, Augath M, Oeltermann A, Logothetis NK. Negative functional MRI response correlates with decreases in neuronal activity in monkey visual area V1. Nat Neurosci. 2006;9(4): 569-77.

116. Biswal B, Yetkin FZ, Haughton VM, Hyde JS. Functional connectivity in the motor cortex of resting human brain using echo-planar MRI. Magn Reson Med. 1995;34(4):537-41.

117. Morgan VL, Gore JC, Abou-Khalil B. Functional epileptic network in left mesial temporal lobe epilepsy detected using resting fMRI. Epilepsy Res.88(2-3):168-78.

118. Pereira FR, Alessio A, Sercheli MS, et al. Asymmetrical hippocampal connectivity in mesial temporal lobe epilepsy: evidence from resting state fMRI. BMC Neurosci. 2010 Jun 2; $11: 66$

119. Bettus G, Guedj E, Joyeux F, et al. Decreased basal fMRI functional connectivity in epileptogenic networks and contralateral compensatory mechanisms. Hum Brain Mapp. 2009;30(5): 1580-91.

120. Bettus G, Bartolomei F, Confort-Gouny S, et al. Role of resting state functional connectivity MRI in presurgical investigation of mesial temporal lobe epilepsy. J Neurol Neurosurg Psychiatry. 2010;81(10):1147-54

121. Moeller F, Maneshi M, Pittau F, et al. Functional connectivity in patients with idiopathic generalized epilepsy. Epilepsia. 2011;52 (3):515-22

122. Bai X, Guo J, Killory B, et al. Resting functional connectivity between the hemispheres in childhood absence epilepsy. Neurology. 2011;76(23):1960-7.

123. Raichle ME, MacLeod AM, Snyder AZ, Powers WJ, Gusnard DA, Shulman GL. A default mode of brain function. Proc Natl Acad Sci USA. 2001;98(2):676-82.

124. Broyd SJ, Demanuele C, Debener S, Helps SK, James CJ, SonugaBarke EJ. Default-mode brain dysfunction in mental disorders: a systematic review. Neurosci Biobehav Rev. 2009;33(3):279-96.

125. Karmonik C, Dulay M, Verma A, Yen C, Grossman RG. Brain activation in complex partial seizures during switching from a the goal-directed task to a resting state: comparison of fMRI maps to the default mode network. Conf Proc IEEE Eng Med Biol Soc. 2010;2010:5685-8.

126. Luo C, Li Q, Lai Y, et al. Altered functional connectivity in default mode network in absence epilepsy: a resting-state fMRI study. Hum Brain Mapp. 2011;32(3):438-49.

127. Petitmengin C, Baulac M, Navarro V. Seizure anticipation: are neurophenomenological approaches able to detect preictal symptoms? Epilepsy Behav. 2006;9(2):298-306.

128. Federico P, Abbott DF, Briellmann RS, Harvey AS, Jackson GD. Functional MRI of the pre-ictal state. Brain. 2005;128(Pt 8): 1811-17.

129. Ebersole JS. Defining epileptogenic foci: past, present, future. J Clin Neurophysiol. 1997;14(6):470-83.

130. Cunningham CJ, Boucousis SM, Goodyear BG, Federico P. Safety and feasibility of using implanted depth electrodes for intracranial EEG-fMRI: a phantom study (abstract). American Epilepsy Society Annual Meeting. 2007;Philadelphia, Pennsylvania.

131. Vulliemoz S, Carmichael DW, Rosenkranz K, et al. Simultaneous intracranial EEG and fMRI of interictal epileptic discharges in humans. Neuroimage. 2011;54(1):182-90. 